\title{
Approaching two decades of cystic fibrosis research in Qatar: a historical perspective and future directions
}

\author{
Samer Hammoudeh', Wessam Gadelhak', Atqah AbdulWahab², Mona Al-Langawi ${ }^{3}$ and Ibrahim A. Janahi ${ }^{2^{*}}$
}

\begin{abstract}
Cystic fibrosis (CF) is a genetic disease caused by a defect of CF transmembrane conductance regulator (CFTR) gene. CF affects multiple systems, predominantly with respiratory involvement. In Qatar, researchers have been exploring various aspects of the disease for almost 20 years. PubMed and Google Scholar were reviewed for articles related to CF in Qatar. The first publication appeared in the year 2000. Since then, several studies have been conducted on CF patients in Qatar considering a variety of topics. The presence of the CFTR I1234V mutation in a certain Arab tribe stands out as a distinguishing characteristic of CF patients in Qatar when compared to the larger Arab region or even worldwide. We aim here to summarize the existing CF research conducted in Qatar over the years as well as to introduce topics for future research.
\end{abstract}

Keywords: Qatar, Cystic fibrosis, Cystic fibrosis transmembrane conductance regulator, CFTR I1234V mutation, Pancreatic sufficient

\section{Background}

Cystic fibrosis (CF) is a multisystem autosomal recessive disease caused by a mutation in the $\mathrm{CF}$ transmembrane conductance regulator (CFTR) gene [1]. Six classes of mutations currently exist, with the most common mutation being F508del, which is classified as a class II mutation [1]. Clinical manifestations include a range of symptoms involving the pulmonary, gastrointestinal, endocrine, and reproductive systems [2]. Morbidity and mortality related to CF are mainly due to respiratory disease [3] and gastrointestinal involvement [4].

CF has been diagnosed to date in a variety of ethnic and racial groups [5]; however, the global epidemiology of CF varies based on a number of factors involving health facilities, clinical awareness, registration systems, and research activities [6]. The prevalence of CF in Northern Europe is reported to be 1 in 2,500 to 3,000, with a carrier rate of 1 in 20 to 30 [5]. Separately, the prevalence of CF among nonHispanic whites, Hispanics, African-Americans, and Asian-

\footnotetext{
* Correspondence: ijanahi@sidra.org

${ }^{2}$ Pediatric Pulmonology, Pediatric Medicine, Sidra Medicine, PO Box 26999,

Doha, Qatar

Full list of author information is available at the end of the article
}

Americans is reported to be 1 in 3,200; 9,200, 15,000, and 31,000 , respectively [5].

In Qatar, the total number of CF patients is 82, including 34 adults (18 males and 16 females) and 48 children (22 males and 26 females). In the pediatric population $(n=48)$, Qataris constitute $62.5 \%(n=30)$ of cases, while non-Qataris constitute $37.5 \%(n=18)$. The majority of these children $65 \%(n=31)$ have the CFTR I1234V mutation, while $35 \%(n=17)$ have other mutations [7]. Based on figures by the Planning and Statistics Authority in Qatar, the population of Qatar is 2,772,294 as of April 2019 [8]. The majority of the population is made of expatriates (88.4\%), as Qatari's represent the remaining $11.6 \%$ of the total population. The majority of the nonQatari population comes from Asia: India 24\%, Nepal 16\%, Philippines 11\%, Bangladesh 5\%, and Sri Lanka 5\%. The gender ration is highly skewed toward males (75\%) which is due to a large influx of male laborers [9].

In the present study, PubMed and Google Scholar were reviewed for articles related to CF in Qatar, using the keywords "cystic fibrosis" AND "Qatar." The references of identified articles were also searched for any additional relevant articles. This paper aims to briefly review the known research related to CF conducted in Qatar and to

(c) The Author(s). 2019 Open Access This article is distributed under the terms of the Creative Commons Attribution 4.0 International License (http://creativecommons.org/licenses/by/4.0/), which permits unrestricted use, distribution, and 
highlight possible future directions in order to attempt to bridge some of the gaps that currently exist in our knowledge, clinical practices, and patient care overall.

\section{The history of cystic fibrosis in Qatar The CFTR I1234V mutation}

In a worldwide analysis, Bobadilla et al. reported that several CFTR mutations are commonly encountered globally; aside from F508del, the list includes G542X, N1303K, and G551D. As for the I1234V mutation, their analysis indicates that it is found in Saudi Arabia and France [10].

The I1234V mutation was first reported in the early 1990s in a sample from southern France [11]. Later on, El-Harith reported the presence of the I1234V mutation in two sisters from Saudi Arabia, who were both homozygous with symptoms of recurrent diarrhea and failure to thrive [12]. Banjar outlined the geographic distribution of mutations in Saudi Arabia and reported that the 1548delG and I1234V mutations are the top two most common CF mutations found among this population [13]. A report by the World Health Organization indicates that the $11234 \mathrm{~V}$ mutation is rarely encountered globally, but is rather frequently encountered in the Middle East among Bedouin tribes [14].

In Qatar, Abdul Wahab et al. in 2001 were the first to report the presence of the homozygous I1234V mutation in patients from 17 families. The study showed that the families in question were from the same Bedouin tribe, with a 96.6\% consanguinity rate, and had varying degrees of clinical manifestations [15]. More than a decade later, Molinski and colleagues examined the variant c.3700A $>$ G in order to study its molecular consequences and found that it causes a splicing mutation rather than a missense mutation, which has initially been predicted to be caused by the variant [16]. More work related to patients in Qatar with the I1234V mutation will be highlighted in the next few sections.

Aside from the I1234V mutation, a few reports have been published to date discussing cases of non-Qataris with other mutations. Abdul Wahab et al. described the case of a Syrian CF child homozygous for the $\Delta \mathrm{F} 508$ mutation with severe clinical manifestations [17]. The 1525-1G > A mutation associated with severe CF was found in a Pakistani child reported on by Abdul Wahab et al. [18]. The N1303K homozygous mutation was detected in an Egyptian child with pseudo-Bartter's syndrome and was reported on in two case reports discussing the various findings $[19,20]$.

\section{Clinical features}

The first publication about CF in Qatar appeared in the year 2000 when Abdul Wahab et al. reported on 45 patients with CF. Qatari children in this study had mild/moderate respiratory symptoms. More severe respiratory symptoms were reported among non-Arabic Asian cases, with an earlier occurrence of Pseudomonas aeruginosa colonization and pancreatic insufficiency as compared with in the aforementioned Qatari cases. The study showed that 26 out of 32 families came from the same Bedouin tribe, with consanguinity reaching a level of $98 \%$ [21].

Later on, Abdul Wahab described the characteristics of a Qatari adult female homozygous for the I1234V mutation. The patient was diagnosed late at the age of 35 years, was multiparous, and had symptoms of chronic lung disease [22]. Then, in 2006, exocrine pancreatic function, using fecal elastase-1, was measured in 40 patients homozygous for the I1234V mutation. This study showed that CF patients were characterized by pancreatic sufficiency. The study also found no difference between using polyclonal antibodies and using monoclonal antibodies when assessing exocrine function [23].

Sweat chloride concentrations [24] and growth parameters as well as calcium homeostasis [25] among $\mathrm{CF}$ patients with the I1234V mutation were investigated by researchers in 2009. Saadoon et al. studied nitric oxide levels among CF patients and found them to have lower fractional exhaled nitric oxide levels in comparison with controls [26]. Another study found a significant association between bone mineral density and forced expiratory volume in $1 \mathrm{~s}$ in a sample of 26 CF patients [27].

Later on, Bhat et al. compared imaging scoring systems as related to the results of pulmonary function testing [28]. Zahraldin and colleagues reported on two siblings with both CF and apparent mineralocorticoid excess and commented on the implications of such [29]. Abdul Wahab et al. studied zinc deficiency in CF patients and concluded that future research into the zinc levels of this patient population as related to Pseudomonas aeruginosa is warranted [30]. More recently, a study comparing adiponectin levels of 17 CF patients with the CFTR I1234V mutation to those of 18 healthy controls found that sputum adiponectin levels could be used to asses inflammatory status among CF patients in a minimally invasive manner [31].

\section{Microbiology}

The study of the microbiology of CF patients in Qatar began in 2004 when Abdul Wahab et al. reported the case of a patient with Achromobacter xylosoxidans [32]. That same year, microbiological patterns of lower airway secretions were described in another paper. Researchers in this study isolated more than 100 pathogens from the samples of $36 \mathrm{CF}$ patients. Typical findings included Pseudomonas aeruginosa, Haemophilus influenza, and Staphylococcus aureus. Less common pathogens were also found, including Mycobacterium abscessus, Stenotrophomonas maltophilia, and Alcaligenes xylosoxidans [33]. 
A few case reports were published during the following year. In the first, Janahi et al. discussed the possibility of a Mycoplasma infection in a female with CF and recommended future investigation of the long-term usage of macrolides [34]. In the second, Abdul Wahab et al. discussed the isolation of Stenotrophomonas maltophilia from a female CF patient with the I1234V mutation [35]. Antimicrobial resistance patterns in bacteria isolates were described by Elshafie et al. in a different paper published in 2007 [36].

Abdul Wahab later on studied the prevalence of Candida dubliniensis [37] and examined the genetic relatedness of Pseudomonas aeruginosa among CF patients and siblings with CF [38] as well as among CF versus non-CF patients [39]. During the following year, researchers compared the accuracy of bacterial identification methods using matrixassisted laser desorption/ionization-time of flight mass spectrometry [40]. Then, in 2017, Abdul Wahab et al. investigated the presence of multidrug-resistant Pseudomonas aeruginosa among CF patients treated with inhaled antibiotics [41]. Separately, authors in another study examined lung function and body mass index in relation to the persistence of Candida dubliniensis [42].

\section{Other related work}

Ben Omran and Abdul Wahab reviewed common genetic disorders in the Qatari population including CF in a book chapter, as part of a book discussing genetic disorders in the Arab world [43]. In 2015, Rehman et al. commented on an article published in The New England Journal of Medicine in regards to the usage of lumacaftor-ivacaftor among CF patients [44]. A few years later, Janahi et al. conducted a review on allergic bronchopulmonary aspergillosis among CF patients [45]. Additionally, Janahi and Rehman published two book chapters, discussing the clinical manifestations and management of CF in the first [46] and presenting a comprehensive review of the CF microbiome in the second [47]. Most recently, Al-Sadeq et al. conducted a review of CF mutations throughout the Arab world [48].

\section{Future directions and recommendations}

\section{Screening programs}

Vast strides have been made in an array of CF-related health care services as well as research activities in Qatar. The introduction of the National Premarital Genetic Screening Program for a number of diseases, including $\mathrm{CF}$, represents one notable milestone that started in 2009. Other diseases screened based on high carrier frequency include: thalassemia, sickle cell disease, classical homocystinuria and spinal muscular dystrophy [49]. Despite the importance of such screening programs, a recent study showed low knowledge and attitudes towards these programs in the Qatari population. The study recommended using educational campaigns at the school and university level [50].

Currently in Qatar, Qatari patients are screened for the founder mutation c.3700 A > G (p.Ile1234Val). Negative cases are tested using the Elucigene CF29 mutation panel, which includes the core panel of $23 \mathrm{CF}$ mutations that are recommended by the American College of Medical Genetics \& Genomics. If no mutation was detected, a full CFTR exon sequencing is conducted.

Molecular carrier screening has been recommended by both the American College of Medical Genetics and Genomics and the American College of Obstetricians and Gynecologists in order for future parents to know their possible risk of conceiving a child with CF. [51] Newborn screening is another route for detection, which has now been implemented across the United States [51]. Moreover, molecular diagnostic testing allows for the earlier identification of cases, which in turn impacts the type of health care services received [51].

\section{Future research and potential therapeutic implications}

The research by Molinski et al. provides invaluable insight into the disease for the local CF population, as related to the phenotype associated with a splicing mutation versus missense mutation, which in turn would reflect on the medical decision-making for these patients. The authors concluded that future treatment modalities are likely to be based on a better understanding of the molecular and clinical aspects related to the variations in CFTR mutations [16]. Building on these conclusions, it would be necessary to conduct genetic sequencing on the remaining CF patients in Qatar as well as their siblings and parents.

Moreover, further studies in the form of observational and experimental research are needed in Qatar. One of the possible future directions for clinical trials is triple-combination therapy, which has been approved by the United States Food and Drug Administration for those who are 12 years of age and older who have two copies of the F508del mutation or who have one mutation that is responsive to tezacaftorivacaftor [52, 53]. Research in other areas including the measurement of patient adherence to medication, dietary habits, and best lifestyle practices remains as topics to be untouched in relation to CF patients in Qatar; thus, intensifying health education efforts regarding these matters also represents another necessary approach.

In the future, establishing and maintaining a registry for CF patients in Qatar is crucial to building a reliable and comprehensive source of information for future research, public health, and health policy objectives [5, 54]. 
Gene editing using clustered regularly interspaced short palindromic repeats (CRISPR) technology is another research area that offers possible promising therapeutic implications, as altering DNA sequences allows for the repair of genetic defects [55]. Relating to this, several scientists have used the CRISPR technique to restore CFTR function, as shown in Schwank et al. [56], Crane et al. [57], Firth et al. [58], or Molinski et al. [59].

The use of organoids is another route for the development or enhancement of personalized medicine in the study of human organ systems $[60,61]$, as it enables the study of human diseases and development by modeling human organs using three-dimensional cultures to proceed [62, 63]. The performance of such research would be beneficial in a unique CF population with the CFTR I1234V mutation.

The role of dietary flavonoids is also an interesting arena for future CF research, as some scientists have suggested that flavonoids may have an impact on patients' immune function and metabolism [64]. Others have hypothesized that flavonoids may have an anti-inflammatory role [65] or might activate the CFTR [66].

\section{Recommendations and conclusion}

As for recommendations, establishing screening panels that are regionally and ethnically specific would help in increasing the detection rate of CF cases [10]. Furthermore, establishing incidence and prevalence statistics for CF-related disorders, locally and also regionally, would aid in future planning and help with determining the necessary size of health education efforts [14]. Finally, planning for the future care of CF patients requires the collaborative efforts of both national and international agencies and/or organizations [54].

In conclusion, the research conducted during the past two decades in Qatar has shed light on several aspects related to CF. However; further research work is necessary and encouraged in the upcoming decades in order to unravel the remaining mysteries associated with this debilitating disease.

\section{Abbreviations}

CF: Cystic fibrosis; CFTR: Cystic fibrosis transmembrane conductance regulator; CRISPR: Clustered regularly interspaced short palindromic repeats

\section{Acknowledgements}

The publication of this article was funded by the Qatar National Library. Contact Details: openaccess@qnl.qa.

\section{Authors' contributions}

SH contributed to the design of the work and drafted the first version, WG: substantively revised it, AA: substantively revised it, MA: substantively revised it, IJ: contributed to the design of the work and substantively revised it. All authors have read and approved the final manuscript.

\section{Funding}

None.
Availability of data and materials

Not applicable.

Ethics approval and consent to participate

Not applicable.

Consent for publication

Not applicable.

\section{Competing interests}

The authors declare that they have no competing interests.

\section{Author details}

${ }^{1}$ Medical Research Center, Research Affairs, Hamad Medical Corporation, PO Box 3050, Doha, Qatar. ${ }^{2}$ Pediatric Pulmonology, Pediatric Medicine, Sidra Medicine, PO Box 26999, Doha, Qatar. ${ }^{3}$ Internal Medicine, Hamad Medical Corporation, PO Box 3050, Doha, Qatar.

Received: 14 February 2019 Accepted: 7 July 2019

Published online: 01 October 2019

\section{References}

1. Elborn JS. Cystic fibrosis. Lancet. 2016;388(10059):2519-31.

2. O'Sullivan BP, Freedman SD. Cystic fibrosis. Lancet. 2009;373(9678):1891-904

3. Ratjen F, Bell SC, Rowe SM, Goss CH, Quittner AL, Bush A. Cystic fibrosis. Nat Rev Dis Primers. 2015;1:15010.

4. Filbrun AG, Lahiri T, Ren CL. Clinical Features and Complications of Cystic Fibrosis. Handbook of Cystic Fibrosis. Cham: Springer International Publishing: 2016. p. 9-41.

5. Filbrun AG, Lahiri T, Ren CL. Introduction and Epidemiology of Cystic Fibrosis. Handbook of Cystic Fibrosis. Cham: Springer International Publishing; 2016. p. 1-4.

6. Mirtajani S, Farnia P, Hassanzad M, Ghanavi J, Farnia P, Velayati A. Geographical distribution of cystic fibrosis; the past 70 years of data analyzis. Biomed Biotechnol Res J. 2017;1(2):105-12.

7. Janahi IA. Assessing the variable molecular \& clinical consequences of the rare CFTR I1234V mutation. Muscat: Regional CF Educational Meeting: Advancing cystic fibrosis total management; 2018.

8. Planning and Statistics Authority (PSA). Statistics overview 2019. Available from: https://www.psa.gov.qa/en/statistics1/Pages/Overview.aspx. [cited 2019 May 30]

9. World Population Review. Qatar Population 2019 2019. Available from: http://worldpopulationreview.com/countries/qatar-population/. [cited 2019 May 30]

10. Bobadilla JL, Macek M, Fine JP, Farrell PM. Cystic fibrosis: A worldwide analysis of CFTR mutations - correlation with incidence data and application to screening. Hum Mutat. 2002;19(6):575-606.

11. Claustres M, Gerrard B, Kjellberg P, Desgeorges M, Demaille J, Dean M. Screening for cystic fibrosis mutations in southern France: identification of a frameshift mutation and two missense variations. Hum Mutat. 1992;1 (4):310-3.

12. el-Harith EA, Dörk T, Stuhrmann M, Abu-Srair H, al-Shahri A, Keller KM, et al. Novel and characteristic CFTR mutations in Saudi Arab children with severe cystic fibrosis. J Med Genet. 1997:34(12):996-9.

13. Banjar H. Geographic distribution of cystic fibrosis transmembrane regulator gene mutations in Saudi Arabia. East Mediterr Health J. 1999;5(6):1230-5.

14. WHO Human Genetics Programme. The molecular genetic epidemiology of cystic fibrosis: report of a joint meeting of WHO/IECFTN/ICF(M)A/ECFS, Genoa, Italy, 19 June 2002. 2004.

15. Abdul Wahab A, Al Thani G, Dawod ST, Kambouris M, Al HM. Heterogeneity of the cystic fibrosis phenotype in a large kindred family in Qatar with cystic fibrosis mutation (11234V). J Trop Pediatr. 2001;47(2):110-2.

16. Molinski SV, Gonska T, Huan L, Baskin B, Janahi IA, Ray PN, et al. Genetic, cell biological, and clinical interrogation of the CFTR mutation c.3700 A>G (p.lle1234Val) informs strategies for future medical intervention. Genet Med. 2014;16(8):625-32

17. Wahab AA, Janahi IA, Hebi S, al-Hamed M, Kambouris M. Cystic fibrosis in a child from Syria. Ann Trop Paediatr. 2002;22(1):53-5.

18. Wahab A, Al Thani G, Dawod ST, Kambouris M, Al HM. Rare CFTR mutation 1525-1G>A in a Pakistani patient. J Trop Pediatr. 2004;50(2):120-2. 
19. Wahab AA, Janahi IA, Marafia MM. An Egyptian Infant with Cystic Fibrosis Mutation N1303K. Qatar Med J. 2004;123:40-1.

20. Wahab AA, Janahi IA, Marafia MM. Pseudo-Bartter's syndrome in an Egyptian infant with cystic fibrosis mutation N1303K. J Trop Pediatr. 2004;50(4):242-4.

21. Abdul Wahab A, Dawod ST, al Thani G. Cystic fibrosis in a large kindred family in Qatar. Ann Trop Paediatr. 2000;20(3):203-7.

22. Wahab AA. Cystic fibrosis mutation 11234 V in a Qatari lady. J Trop Pediatr. 2003:49(1):54-5.

23. Abdel Rahman H, Abdul Wahab A, Abdel Rahman MO, Mostafa OA. Faecal elastase-1 concentration in cystic fibrosis patients with CFTR $11234 \mathrm{~V}$ mutation. Acta Paediatr. 2006;95(9):1066-9.

24. Abdul-Wahab A, Janahi IA, Abdel-Rahman MO. Sweat chloride concentration in cystic fibrosis patients with cystic fibrosis trans-membrane conductance regulator I1234V mutation. Saudi Med J. 2009;30(8):1101-2

25. Wahab AA, Soliman A, Rahman MOA. Growth parameters and calcium homeostasis in cystic fibrosis patients with CFTR I1234V mutation. Ann Saudi Med. 2009:29(6):487-8.

26. Saadoon A, Janahi I, Abdul-Wahab A, Al-Langawi M. Baseline Exhaled Nitric Oxide Level In Cystic Fibrosis Patients With I1234V Mutation Is Lower Than Normal And Affected By Gender And Airway Microbiome. San Diego: D25 Pediatric Cystic Fibrosis; 2014. p. A5518-A.

27. Abdul Wahab A, Hammoudeh M, Allangawi M, Al-Khalaf F, Chandra P. Bone mineral density in cystic fibrosis patients with the CFTR I1234V mutation in a large kindred family is associated with pancreatic sufficiency. Int J Rheumatol. 2014:2014:465395.

28. Bhat V, Wahab AA, Garg KC, Janahi I, Singh R. HRCT in cystic fibrosis in patients with CFTR I1234V mutation: assessment of scoring systems with low dose technique using multidetector system and correlation with pulmonary function tests. Indian J Radiol Imaging. 2015;25(1):44-51.

29. Zahraldin K, Janahi IA, Ben-Omran T, Alsulaiman R, Hamad B, Imam A. Two Qatari siblings with cystic fibrosis and apparent mineralocorticoid excess. Ann Thorac Med. 2015;10(1):69-72.

30. AbdulWahab A, Abushahin A, Allangawi M, Chandra P, Abdel Rahman MO, Soliman A. Serum zinc concentration in cystic fibrosis patients with CFTR I1234V mutation associated with pancreatic sufficiency. Clin Respir J. 2017; 11(3):305-10.

31. Abdul Wahab A, Allangawi M, Thomas M, Bettahi I, Silvaraman S, Kumar J, et al. Sputum and plasma adiponectin levels in clinically stable adult cystic fibrosis patients with CFTR I1234V mutation. Eur Respir J. 2018;52(suppl 62): PA1334.

32. Abdul Wahab A, Janahi IA, El-Shafie SS. Achromobacter xylosoxidans isolated from the sputum of a patient with cystic fibrosis mutation I1234V with Pseudomonas aeruginosa. Saudi Med J. 2004;25(6):810-1.

33. Wahab AA, Janahi IA, Marafia MM, El-Shafie S. Microbiological identification in cystic fibrosis patients with CFTR I1234V mutation. J Trop Pediatr. 2004; 50(4):229-33.

34. Janahi IA, Abdulwahab A, Elshafie Sittana S, Bush A. Rapidly progressive lung disease in a patient with cystic fibrosis on long-term azithromycin: possible role of mycoplasma infection. J Cystic fibros. 2005:4(1):71-3.

35. Abdul Wahab A, Janahi I, Elshafie S. Stenotrophomonas Maltophilla Isolated from the Sputum of the Patient with Cystic Fibrosis Mutation 11234V. First Qatari Report. 2005;14:57-8.

36. Elshafie SS, Wahab AA, Janahi IA. Antimicrobial resistance of bacterial strains isolated from respiratory tract of cystic fibrosis patients with CFTR I1234V mutation. J Pediatr Infect Dis. 2007:02(01):039-43.

37. Wahab AA, Taj-Aldeen SJ, Kolecka A, ElGindi M, Finkel JS, Boekhout T. High prevalence of Candida dubliniensis in lower respiratory tract secretions from cystic fibrosis patients may be related to increased adherence properties. Int J Infect Dis. 2014;24:14-9.

38. Abdul Wahab A, Taj-Aldeen SJ, Hagen F, Diophode S, Saadoon A, Meis $\mathrm{JF}$, et al. Genotypic diversity of Pseudomonas aeruginosa in cystic fibrosis siblings in Qatar using AFLP fingerprinting. Eur J Clin Microbiol Infect Dis. 2014;33(2):265-71.

39. AbdulWahab A, Taj-Aldeen SJ, Ibrahim E, Abdulla SH, Muhammed R, Ahmed I, et al. Genetic relatedness and host specificity of Pseudomonas aeruginosa isolates from cystic fibrosis and non-cystic fibrosis patients. Infect Drug Resist. 2014;7:309-16.

40. AbdulWahab A, Taj-Aldeen SJ, Ibrahim EB, Talaq E, Abu-Madi M, Fotedar R. Discrepancy in MALDI-TOF MS identification of uncommon gram-negative bacteria from lower respiratory secretions in patients with cystic fibrosis. Infect Drug Resist. 2015;8:83-8.
41. AbdulWahab A, Zahraldin K, Sid Ahmed MA, Jarir SA, Muneer M, Mohamed $\mathrm{SF}$, et al. The emergence of multidrug-resistant Pseudomonas aeruginosa in cystic fibrosis patients on inhaled antibiotics. Lung India. 2017;34(6):527-31.

42. AbdulWahab A, Salah H, Chandra P, Taj-Aldeen SJ. Persistence of Candida dubliniensis and lung function in patients with cystic fibrosis. BMC Res Notes. 2017;10(1):326.

43. Ben-Omran T, Abdul W. Genetic disorders in Arab populations: Qatar. Genetic Disorders in the Arab World, Qatar. 42012. p. 60-5.

44. Rehman A, Baloch NU, Janahi IA. Lumacaftor-Ivacaftor in patients with cystic fibrosis homozygous for Phe508del CFTR. N Engl J Med. 2015; 373(18):1783.

45. Janahi IA, Rehman A, Al-Naimi AR. Allergic bronchopulmonary aspergillosis in patients with cystic fibrosis. Ann Thorac Med. 2017;12(2):74-82.

46. Janahi I, Rehman A. Clinical manifestations of cystic fibrosis and their management. In: Robertson L, editor. Cystic and idiopathic pulmonary fibrosis: risk factors, Management and Long-Term Health Outcomes. New York: Nova Science Publishers; 2016. p. 1-56.

47. Janahi IA, Rehman A. The cystic fibrosis airway microbiome and pathogens. In: Sriramulu D, editor. Progress in Understanding Cystic Fibrosis. London: IntechOpen; 2017

48. Al-Sadeq D, Abunada T, Dalloul R, Fahad S, Taleb S, Aljassim K, et al. Spectrum of mutations of cystic fibrosis in the 22 Arab countries: A systematic review. Respirology. 2018;24(2):127-36.

49. Al-Dewik N, Al-Mureikhi M, Shahbeck N, Ali R, Al-Mesaifri F, Mahmoud L, et al. Clinical genetics and genomic medicine in Qatar. Mol Genet Genomic Med. 2018;6(5):702-12

50. Bener A, Al-Mulla M, Clarke A. Premarital screening and genetic counseling program: studies from an endogamous population. Int J Appl Basic Med Res. 2019;9(1):20-6.

51. Brennan M-L, Schrijver I. Cystic fibrosis: A review of associated phenotypes, use of molecular diagnostic approaches, genetic characteristics, Progress, and dilemmas. J Mol Diagn. 2016;18(1):3-14.

52. Inacio P. FDA approves Symdeko, Vertex's combo therapy for patients with certain mutations in CFTR gene. 2018. Available from: https:// cysticfibrosisnewstoday.com/2018/02/13/fda-approves-symdekotmtezacaftor-ivacaftor-to-treat-cystic-fibrosis-patients-with-certain-cftrmutations/. [cited 2018 October 21].

53. Kunzmann K. FDA Approves Triple Combination Therapy for Cystic Fibrosis 2018. Available from: https://www.mdmag.com/medical-news/fda-approvestriple-combination-therapy-for-cystic-fibrosis. [cited 2018 October 21].

54. WHO HGP. Implementation of cystic fibrosis services in developing countries: report of a joint WHO/international cystic fibrosis (Mucoviscidosis) association meeting. 1995

55. Marangi M, Pistritto G. Innovative therapeutic strategies for cystic fibrosis: moving forward to CRISPR technique. Front Pharmacol. 2018;9:396.

56. Schwank G, Koo BK, Sasselli V, Dekkers JF, Heo I, Demircan T, et al. Functional repair of CFTR by CRISPR/Cas9 in intestinal stem cell organoids of cystic fibrosis patients. Cell Stem Cell. 2013;13(6):653-8.

57. Crane AM, Kramer P, Bui JH, Chung WJ, Li XS, Gonzalez-Garay ML, et al. Targeted correction and restored function of the CFTR gene in cystic fibrosis induced pluripotent stem cells. Stem Cell Rep. 2015;4(4):569-77.

58. Firth AL, Menon T, Parker GS, Qualls SJ, Lewis BM, Ke E, et al. Functional gene correction for cystic fibrosis in lung epithelial cells generated from patient iPSCs. Cell Rep. 2015;12(9):1385-90.

59. Molinski SV, Ahmadi S, Ip W, Ouyang H, Villella A, Miller JP, et al. Orkambi(R) and amplifier co-therapy improves function from a rare CFTR mutation in gene-edited cells and patient tissue. EMBO Mol Med. 2017;9(9):1224-43.

60. Noordhoek J, Gulmans V, van der Ent K, Beekman JM. Intestinal organoids and personalized medicine in cystic fibrosis: a successful patient-oriented research collaboration. Curr Opin Pulm Med. 2016;22(6):610-6.

61. Hohwieler M, Perkhofer L, Liebau S, Seufferlein T, Müller M, Illing A, et al. Stem cell-derived organoids to model gastrointestinal facets of cystic fibrosis. United European Gastroenterol J. 2017;5(5):609-24.

62. Simian M, Bissell MJ. Organoids: A historical perspective of thinking in three dimensions. J Cell Biol. 2017;216(1):31-40.

63. Lancaster MA, Knoblich JA. Organogenesis in a dish: Modeling development and disease using organoid technologies. Science. 2014; 345(6194):1247125.

64. Li L, Somerset S. Associations between Flavonoid Intakes and Gut Microbiota in a Group of Adults with Cystic Fibrosis. Nutrients. 2018;10(9): 1264-76. 
65. Nori SL, Aquino RP, Nicolin V, Santoro A. Flavonoids and flavonoidrich natural extracts inhibit cytokine release in cystic fibrosis bronchial epithelial cells by regulating NF-kB pathway. Ital J Anat Embryol. 2015:120(1):53.

66. Pyle LC, Fulton JC, Sloane PA, Backer K, Mazur M, Prasain J, et al. Activation of the cystic fibrosis transmembrane conductance regulator by the flavonoid quercetin: potential use as a biomarker of $\Delta$ F508 cystic fibrosis transmembrane conductance regulator rescue. Am J Respir Cell Mol Biol. 2010;43(5):607-16.

\section{Publisher's Note}

Springer Nature remains neutral with regard to jurisdictional claims in published maps and institutional affiliations.

Ready to submit your research? Choose BMC and benefit from:

- fast, convenient online submission

- thorough peer review by experienced researchers in your field

- rapid publication on acceptance

- support for research data, including large and complex data types

- gold Open Access which fosters wider collaboration and increased citations

- maximum visibility for your research: over $100 \mathrm{M}$ website views per year

At $B M C$, research is always in progress.

Learn more biomedcentral.com/submissions 